\title{
Iterative Algorithm of Atomic Potential Reconstruction Based on DPC Signal from Thick Specimens
}

\author{
Takehito Seki ${ }^{1}$, Yuichi Ikuhara ${ }^{1,2}$ and Naoya Shibata ${ }^{1,2^{*}}$ \\ 1. Institute of Engineering Innovation, School of Engineering, The University of Tokyo, Tokyo, Japan \\ 2. Nanostructure Research Laboratory, Japan Fine Ceramic Center, Nagoya, Japan \\ * Corresponding author: shibata@ sigma.t.u-tokyo.ac.jp
}

In scanning transmission electron microscopy (STEM), electromagnetic fields inside specimens can be measured by detecting deflection of the convergent electron beam using a segmented or pixelated detector placed in the diffraction plane. This imaging technique is called differential phase contrast (DPC). Electric field measurement can be carried out at atomic resolution, and electric field between nuclei and electrons has been visualized [1-4]. According to Maxwell's equation, static electric field can be converted into charge density map. This conversion was demonstrated at atomic resolution, and electron cloud surrounding nuclei has been visualized [5]. If the electron density mapping can be performed more accurately, DPC STEM potentially enables direct imaging of chemical bonding at crystal defects: e.g., surfaces, interfaces, dislocations and point defects. However, accurate quantification of electric field in thick specimens is still challenging because of the dynamical effect. In this study, we aim to develop an iterative algorithm to overcome the dynamical effect and reconstruct accurate atomic potential on the basis of DPC signal.

For thin specimens such as monoatomic structure, an accurate reconstruction technique has been developed on the basis of phase contrast transfer function (PCTF) combined with detecting the first moment of the diffraction intensity [6]. This technique is accurate even for heavy elements because the first-moment detection concept is valid within the phase object approximation $[7,8]$. In thick specimens, this technique does not work accurately, since a typical specimen is thicker than the depth of field of the aberration-corrected STEM probe. Thus, probe propagation always should be taken into account even if specimen potential is weak enough to neglect multiple scattering. Under the first Born approximation, a new type of PCTF, integrated PCTF (iPCTF), can be defined for thick specimens by integrating the conventional PCTF along thickness direction. Validity of iPCTF has been demonstrated through explaining imaging characteristic of annular bright field [9]. Furthermore, iPCTF can explain focal dependence of DPC imaging; maximum contrast can be obtained with a probe focused on the mid-plane of specimens. Therefore, we adopt iPCTF combined with the first-moment detection concept as a linear imaging model in the reconstruction algorithm.

To overcome the multiple-scattering effect, multi-slice simulation is further combined in the iterative algorithm. Initially, specimen potential is estimated from an experimental DPC image through the iPCTF theory, and multi-slice simulation is carried out on the basis of the estimated potential. A residual DPC image is calculated by subtracting the simulated image from the experimental image. The residual image is generally not negligible due to inaccuracy of the first Born approximation. The estimated potential is modified on the basis of the residual DPC image through iPCTF theory, and then, multi-slice simulation is carried out again. This procedure is iteratively repeated until the residual becomes sufficiently small.

Validity of the newly developed algorithm was checked by reconstructing potential from simulated DPC 
images. Fig. 1 shows simulated DPC images of Si along [110] with thickness of $11 \mathrm{~nm}$ and defocus of $5.4 \mathrm{~nm}$. Since the simulated images are affected by multiple scattering, atomic potential reconstructed within the first Born approximation is apparently inaccurate (Fig. 2a). The initial residual factor from the true potential within the information limit is $114 \%$. Contrastingly, iteratively reconstructed potential appears to be much more accurate (Fig. 2b), and its residual factor is less than $0.5 \%$. Details and experimental results will be discussed in the presentation.

\section{References:}

[1] N. Shibata et al., Nature Physics 8 (2012) 611.

[2] N. Shibata et al., Nature Communications 8 (2017) 15631.

[3] R. Ishikawa et al., Nature Communications 9 (2018) 3878.

[4] N. Shibata et al., Accounts of Chemical Research 50 (2017) 1502.

[5] G. Sánchez-Santolino et al., ACS Nano 12 (2018) 8875.

[6] T. Seki et al., Ultramicroscopy 182 (2017) 258.

[7] E. Waddell et al., Optik 54 (1979) 83.

[8] K. Müller et al., Nature Communications 5 (2014) 5653.

[9] T. Seki, N. Takanashi and E. Abe, Ultramicroscopy 194 (2018) 193.

[10] This work was supported by the SENTAN, Japan Science and Technology Agency, and the JSPS KAKENHI Grant number JP17H01316.
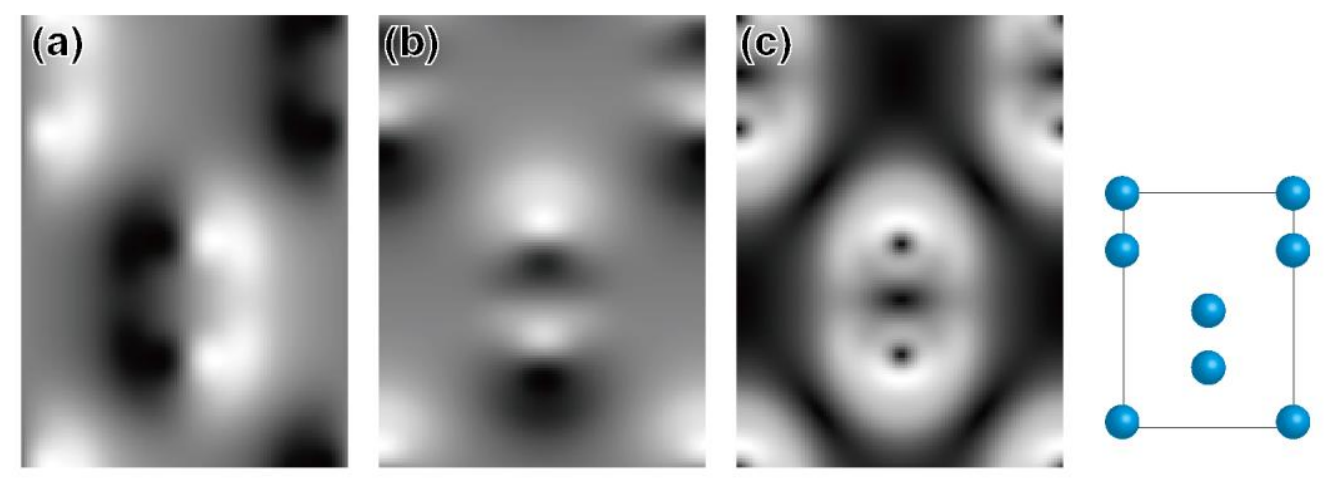

Figure 1. (a) Lateral component, (b) vertical component, and (c) modulus of simulated atomic electric field perpendicular to $\mathrm{Si}$ [110] axis.
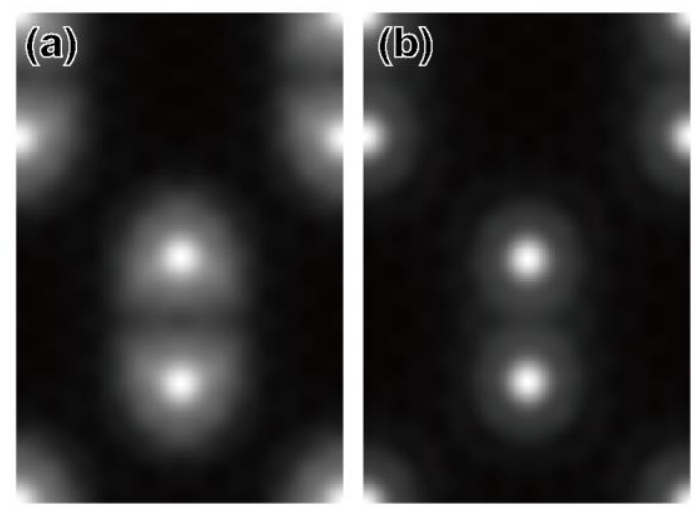

Figure 2. (a) Initially reconstructed potential and (b) iteratively reconstructed potential of Si [110]. 